\title{
Control of Distributed Embedded Systems in the Presence of Unknown-but-Bounded Noise
}

B.Picasso

L.Palopoli

Abstract-In this paper we consider the problem of controlling multiple scalar systems through a limited capacity shared channel. Each system is affected by process noise and can be controlled by actuators with values in a fixed finite set. The control objective is to bound the evolution of the systems in specified sets (controlled invariance). Our goal is to find an optimal allocation of the shared communication resource to the different control activities and to identify correct choices for the design parameters. The paper provides fundamental conceptual tools to attack the design problem in the formal framework of an optimization problem. Namely, we give a feasibility criterion to decide whether a set of design parameters conforms with a control specification (i.e., with the controlled invariance of a specified set for each system). Moreover, we offer the explicit computation of the minimum bit rate necessary for the controlled invariance of a set, which is of utmost importance for solving the optimization problem.

\section{INTRODUCTION}

Traditional control design is based on ideal assumptions concerning the amount, type and accuracy of the information flow that can be circulated across the controller. Unfortunately, real implementation platforms exhibit nonidealities that may substantially invalidate such assumptions. As a result, the system's closed-loop performance can be severely affected and sometimes stability itself is jeopardized. These problems show up with particular strength when multiple control loops share a limited pool of computation and communication resources. In this case the designer is confronted with the challenging task of choosing at the same time the control law and the optimal allocation policy for the shared resources (control algorithm/system architecture co-design). An intriguing general discussion for this class of problems can be found in [5]. Investigations in this field have been developed in several directions. A first prong of research activities has focused on the problem of resource sharing [16], [12], [7]. However, these papers do not explicitly cope with quantization and bit rate constraints that play an important role in complex distributed systems. A remarkable thread of papers has focused on the problem

This work was supported by European Commission through the IST2001-37170 RECSYS project, by MIUR PRIN 095297_002-2002 and by the Swedish Research Council.

B.Picasso (corresponding author) is with "Scuola Normale Superiore", Pisa. e-mail: b.picasso@sns. it

L.Palopoli is with "Scuola Superiore S.Anna, ReTiS Lab", Pisa. e-mail: palopoliasssup.it

A.Bicchi is with "University of Pisa, Centro E. Piaggio". e-mail: bicchi@ing.unipi.it

K.H.Johansson is with "Royal Institute of Technology, KTH", Stockholm. e-mail: kallejakth. se of stabilization under bit rate constraints [8], [19], [9], [15], [10], [14], [2]. In these works, the authors generally synthesize quantization schemes instrumental to the goal of finding encoding-decoding policies that make for an optimal use of the channel, when the latter is used in a control loop. Albeit interesting from a theoretical point of view, this approach is to be verified from the standpoint of technological feasibility. In [17], [18] a different view is taken. The authors analyze the attainable control performance when quantization is a fixed element of the problem. An evident motivation for this work is the analysis of control systems where actuation and/or sensing are inherently quantized, e.g., stepper motors, D/A converters, quantized encoders.

In this work, we make the same assumption: control loops are operated by quantized actuators, which are regarded as given "hardware" components to build on the top of. The general setting we refer to is depicted in Fig. 1: a limited

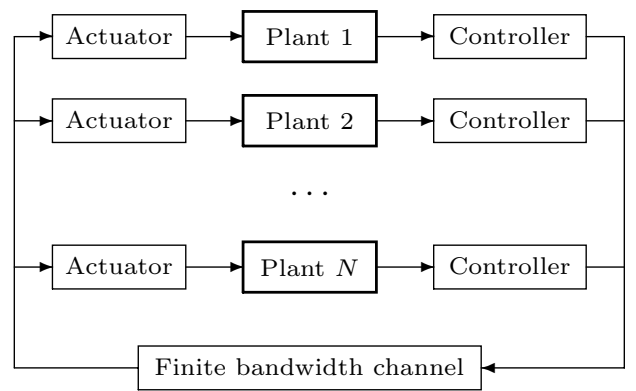

Fig. 1. Pictorial representation of the problem analyzed in the paper.

bandwidth channel is shared between several independent control loops. This can be thought of as "smart-sensor" scenario, i.e., one where processing activities are located in the proximity of sensors and commands have to be sent to actuators by a channel. For the sake of simplicity and as a first step towards a more general theory, we restrict the analysis to scalar systems ruled by control laws generated by periodic sampling and consider uniform quantizers. In order to limit the bandwidth used for the transmission, some of the levels provided by the quantized controller can be left unused. Therefore the subset $\mathcal{U}$ necessary to accomplish the control task is a design parameter along with the sampling period. Our final goal is to produce automated procedures for the optimal allocation of the channel capacity among the different loops and for the choice of the set of design parameters. Both problems require a thorough 
investigation of the design problem for a single loop, which is the main contribution of this paper. We will address two fundamental questions: the identification of the feasible design parameters with respect to a control specification and the determination of the minimum bit rate necessary to attain a specification. As far as the control goal is concerned, it is well known [8] that classical notion of stability has evident shortcomings when quantization is in place. We hence aim at "practical" stability. In particular, in this paper we are concerned with the steady-state behavior of the system, i.e., the ability to make a desired set invariant. This concept is closely related to the notion of "boundability" [2]. The presence of noise is of particular interest in our context, while this problem has not been extensively regarded in the previous literature on quantized systems. Indeed, the presence of noise emphasizes the importance of the sampling period especially for unstable systems since long sampling intervals determine performance degradation due to longer uncompensated actions of the noise term.

The paper is organized as follows. In Sec. II we formally introduce the problem dealt with in the paper. In Sec. III the analysis for a single plant is developed and the basic tools to face the multi-plant problem are derived. Some conclusions and open problems are suggested in Sec. IV .

Notation: $I(\Lambda):=\left[-\frac{\Lambda}{2} ; \frac{\Lambda}{2}\right] .\lfloor x\rfloor:=\max \{z \in \mathbb{Z} \mid z \leq$ $x\}$ and $\lceil x\rceil:=\min \{z \in \mathbb{Z} \mid z \geq x\}$ are the floor and the ceil functions. The Lebesgue measure is denoted by $\lambda(\cdot)$.

\section{PROBLEM FORMULATION}

Consider a set of $N \geq 1$ scalar plants

$$
\left\{\begin{array}{l}
\dot{\tilde{x}}_{i}(t)=a_{i} \tilde{x}_{i}(t)+\tilde{u}_{i}(t)+\tilde{w}_{i}(t) \\
\tilde{x}_{i}(0)=\tilde{x}_{i}^{0} \\
i=1, \ldots, N
\end{array}\right.
$$

where the control function $\tilde{u}_{i}(t)$ takes values in a regularly quantized set $\mathcal{U}_{i} \subseteq \epsilon_{i} \mathbb{Z}\left(\epsilon_{i}>0\right)$ and $\tilde{w}_{i}(t) \in I\left(\mathrm{w}_{i}\right)$ $\left(\mathrm{w}_{i} \geq 0\right)$ represents an exogenous noise term. It is supposed that $\tilde{w}_{i}(t)$ is an integrable function. Each scalar system is denoted by the triple $\left(a_{i}, \epsilon_{i}, \mathrm{w}_{i}\right)$ which are the given parameters characterizing the system.

We assume that the states $\tilde{x}_{i}(i=1, \ldots, N)$ are sampled periodically at time $0, T_{i}, 2 T_{i}, \ldots$ (the sampling intervals $T_{i}$ are design parameters). Based on these samples, $N$ individual control values are derived and transmitted over a shared communication channel to zero-order hold devices in the corresponding actuator nodes. The sampled-data control system corresponding to (1) is

$$
\left\{\begin{array}{l}
x_{i}(k+1)=\Phi_{i} x_{i}(k)+\Gamma_{i} u_{i}(k)+w_{i}(k) \\
x_{i}(0)=\tilde{x}_{i}^{0} \\
i=1, \ldots, N
\end{array}\right.
$$

where $x_{i}(k)=\tilde{x}_{i}\left(k T_{i}\right)$ and

$$
\begin{aligned}
\Phi_{i} & =e^{a_{i} T_{i}} \\
\Gamma_{i} & =\int_{0}^{T_{i}} e^{a_{i} s} d s \\
w_{i}(k) & =\int_{k T_{i}}^{(k+1) T_{i}} e^{a_{i}\left((k+1) T_{i}-s\right)} \tilde{w}_{i}(s) d s .
\end{aligned}
$$

Each $T_{i}$-sampled system is denoted by the quadruple $\left(a_{i}, \epsilon_{i}, \mathrm{w}_{i}, T_{i}\right)$. From the last equation it follows that the discrete time disturbance $w_{i}(k)$ takes values in $I\left(\Gamma \cdot \mathrm{w}_{i}\right)$. In this setting the quantized discrete time control law is a function

$$
\begin{aligned}
q_{i}: \mathbb{R} & \rightarrow \mathcal{U}_{i} \subseteq \epsilon_{i} \mathbb{Z} \\
x_{i}(k) & \mapsto u_{i}(k)
\end{aligned}
$$

so that with the zero-order hold the continuous time control law is the piecewise constant function

$$
\tilde{u}_{i}(t)=u_{i}(k), \quad t \in\left[k T_{i} ;(k+1) T_{i}[.\right.
$$

The plants share a limited bandwidth channel with bit rate $\mathcal{R}$, hence the bit rates $R_{i}$ 's devoted to each control loop, which will be allocated on the basis of some optimality criterion, have to comply with the following inequality:

$$
\sum_{i=1}^{N} R_{i} \leq \mathcal{R}
$$

The target is to guarantee "practical" stability for each plant, more precisely we consider the controlled invariance problem. Namely, we are interested in finding neighborhoods of the equilibrium where the trajectories of each plant can be confined irrespective to any noise affecting the systems. In our framework we refer to the following definitions of controlled invariance (see also [4]):

Definition 1 (Continuous time): For a system $(a, \epsilon, \mathrm{w})$ with control set $\mathcal{U}$, the interval $I(\Delta)$ is said to be controlled invariant iff there exists $T>0$ such that $\forall \tilde{x}^{0} \in I(\Delta)$ there exists $u \in \mathcal{U}$ such that for any integrable function $\tilde{w}:[0 ; T] \rightarrow I(\mathrm{w})$ the solution of

$$
\left\{\begin{array}{l}
\dot{\tilde{x}}(t)=a \tilde{x}(t)+u+\tilde{w}(t) \\
\tilde{x}(0)=\tilde{x}^{0}
\end{array}\right.
$$

is $\tilde{x}(t) \in I(\Delta) \forall t \in[0 ; T]$.

Definition 2 (Discrete time): For a system $(a, \epsilon, \mathrm{w}, T)$ with control set $\mathcal{U}$, the interval $I(\Delta)$ is said to be controlled invariant iff $\forall x^{0} \in I(\Delta)$ there exists $u \in \mathcal{U}$ such that $\forall w \in I(\Gamma \cdot \mathrm{w}), x^{0+}=\Phi x^{0}+\Gamma u+w \in I(\Delta)$. The following proposition allows us to get rid of the distinction between continuous time and discrete time:

Proposition 1: Consider the $T$-sampled control system $(a, \epsilon, \mathrm{w}, T)$ : if $x^{0} \in I(\Delta)$ and $u \in \mathcal{U}$ is such that $x^{0+}=\Phi x^{0}+\Gamma u+w \in I(\Delta) \forall w \in I(\Gamma \cdot \mathrm{w})$, then for any integrable function $\tilde{w}:[0 ; T] \rightarrow I(\mathrm{w})$ the solution $\tilde{x}(t)$ of

$$
\left\{\begin{array}{l}
\dot{\tilde{x}}(t)=a \tilde{x}(t)+u+\tilde{w}(t) \\
\tilde{x}(0)=x^{0}
\end{array}\right.
$$

is such that $\tilde{x}(t) \in I(\Delta) \forall t \in[0 ; T]$.

Therefore, if $I(\Delta)$ is controlled invariant for the discrete time system $(a, \epsilon, \mathrm{w}, T)$, then it is controlled invariant for the continuous time system $(a, \epsilon, \mathrm{w})$.

Proof: See Appendix.

Conversely it is obvious that if $I(\Delta)$ is controlled invariant for system $(a, \epsilon, \mathrm{w})$, then $I(\Delta)$ is controlled invariant for system $(a, \epsilon, \mathrm{w}, T)$ for any $T$ as in Definition 1 . Thus, it 
will be sufficient to introduce and to check the properties based on invariance only for discrete time models.

The channel: By a limited bandwidth channel of capacity $\mathcal{R}$ we mean a device capable of transmitting $\mathcal{R}$ bits per unit of time. In particular, the number of symbols $\sigma$ that can be transmitted during the time interval $T$ satisfies $\sigma \leq 2^{\mathcal{R} T}$. Since the number of bits to be transmitted at each sampling instant is integer, we require that $\sigma \leq 2^{\lfloor\mathcal{R} T\rfloor}$.

Definition 3: Consider system $(a, \epsilon, \mathrm{w})$, suppose that a channel of capacity $R$ is connecting the controller to the plant: the triple $(R, T, \Delta)$ is said to be feasible for the invariance problem iff there exists a control set $\mathcal{U} \subset \epsilon \mathbb{Z}$ rendering $I(\Delta)$ controlled invariant for system $(a, \epsilon, \mathrm{w}, T)$ and satisfying $\# \mathcal{U} \leq 2^{\lfloor R T\rfloor}$.

Consider the set of systems $\left(a_{i}, \epsilon_{i}, \mathrm{w}_{i}\right), i=1, \ldots, N$. Let $\vec{R}:=\left(R_{1}, \ldots, R_{N}\right), \vec{T}:=\left(T_{1}, \ldots, T_{N}\right)$ and $\vec{\Delta}:=$ $\left(\Delta_{1}, \ldots, \Delta_{N}\right)$. The triple $(\vec{R}, \vec{T}, \vec{\Delta})$ is said to be feasible iff $\forall i=1, \ldots, N,\left(R_{i}, T_{i}, \Delta_{i}\right)$ is feasible for the invariance problem related to system $\left(a_{i}, \epsilon_{i}, \mathrm{w}_{\mathrm{i}}\right)$.

The presence of multiple plants opens up different design possibilities as to how the communication capacity of the link can be shared between the different control loops. As a first example, it is reasonable to require that the measure $\Delta_{i}$ of the invariant set attained on the $i$-th loop be in a half-space $\Delta_{i} \leq \bar{\Delta}_{i}$. More generally, we require that $\vec{\Delta}$ be in a specified domain: $\vec{\Delta} \in \mathcal{D}$. The design problem can be formulated as follows:

$$
\begin{gathered}
\min _{(\vec{R}, \vec{T}, \vec{\Delta})} f(\vec{\Delta}) \\
\text { subj. to: }\left\{\begin{array}{l}
\overrightarrow{\vec{\Delta} \in \mathcal{D}} \\
\sum_{i=1}^{N} R_{i} \leq \mathcal{R} \\
(\vec{R}, \vec{T}, \vec{\Delta}) \text { feasible }
\end{array}\right.
\end{gathered}
$$

where $f$ is a cost function. Setting $f$ to 0 amounts to formulating a feasibility problem, i.e., the search for a feasible solution within the specified constraints. A different choice can be to relate $f$ to the attainment of a specified vector $\vec{\Delta}_{0}$. Hence, $f$ must penalize realizations $\vec{\Delta}$ differing from the desired target. E.g., $f(\vec{\Delta}):=\frac{\left\|\vec{\Delta}_{0}-\vec{\Delta}\right\|}{\left\|\vec{\Delta}_{0}\right\|}$.

Let $R_{\min }(\vec{\Delta}):=\sum_{i=1}^{N} R_{\min }^{i}\left(\Delta_{i}\right)$, where $R_{\min }^{i}\left(\Delta_{i}\right)$ is the smallest bit rate $R_{i}$ ensuring that there exists a choice of $T_{i}$ such that $\left(R_{i}, T_{i}, \Delta_{i}\right)$ is feasible. It is immediate to check that the minimum of problem (4) is equal to

$$
\begin{gathered}
\min _{\vec{\Delta}} f(\vec{\Delta}) \\
\text { subj. to: }\left\{\begin{array}{l}
\vec{\Delta} \in \mathcal{D} \\
R_{\min }(\vec{\Delta}) \leq \mathcal{R}
\end{array}\right.
\end{gathered}
$$

Therefore, the solution of the problem is significantly aided by the availability of the function $R_{\min }^{i}(\Delta)$ for each plant. For instance the feasibility problem becomes almost straightforward if such a function is available. Thereby, the computation of this function is one of the most relevant contribution of the paper.

We end this section with an example to be regarded as a aid for the understanding of the problem setting.
Example 1 (Tracking of an unknown reference): Consider $N$ agents moving on a line according to $\dot{\tilde{y}}_{i}(t)=\tilde{u}_{i}(t)$, $i=1, \ldots, N$. Let $r(t)$ be an unknown reference to track and assume that $|\dot{r}(t)| \leq \frac{\mathrm{w}}{2}$. A camera takes the measures of the displacements $\tilde{e}_{i}(t):=\tilde{y}_{i}(t)-r(t)$ of the agents and sends the quantized control values $\tilde{u}_{i}$ 's to the actuators through a shared channel of capacity $\mathcal{R}$. The resulting dynamics of the displacement of the $i$-th agent is $\dot{\tilde{e}}_{i}(t)=\tilde{u}_{i}(t)-\dot{r}(t)$ so that the problem is modelled by system (1) with $a_{i}=0 \forall i$.

\section{Single PLANT ANALYSIS}

This section is devoted to the characterization of feasible triples $(R, T, \Delta)$ and to the calculation of $R_{\min }(\Delta)$.

\section{A. Feasibility analysis}

Given $\Delta>0$ and $T>0$, suppose that there exists a control set $\mathcal{U} \subset \epsilon \mathbb{Z}$ rendering $I(\Delta)$ controlled invariant for system $(a, \epsilon, \mathrm{w}, T)$. Let $\ell(\Delta, T) \in \mathbb{N}$ be the minimum of the cardinality of the control sets $\mathcal{U} \subset \epsilon \mathbb{Z}$ rendering $I(\Delta)$ controlled invariant.

Theorem 1 (Feasibility test): Consider system $(a, \epsilon, \mathrm{w})$, the triple $(R, T, \Delta)$ is feasible if and only if

$$
R \geq \frac{1}{T}\left\lceil\log _{2} \ell(\Delta, T)\right\rceil
$$

Proof: It is an immediate consequence of the definitions of feasibility and of the function $\ell(\Delta, T)$.

Since we will be able to determine the expression of the function $\ell(\Delta, T)$, Theorem 1 will be an effective tool to solve the feasibility problem.

Let us start by the characterization of the domain of $\ell(\Delta, T)$. Given $\Delta>0$, assume that $\mathcal{U}=\epsilon \mathbb{Z}$ and let

$$
\begin{aligned}
& \mathcal{T}(\Delta):=\{T>0 \mid I(\Delta) \text { is controlled invariant } \\
& \text { for system }(a, \epsilon, \mathrm{w}, T)\} \text {. }
\end{aligned}
$$

The domain of $\ell(\Delta, T)$ is $\{(\Delta, T) \mid T \in \mathcal{T}(\Delta)\}$.

We hence start with the determination of the set $\mathcal{T}(\Delta)$. The main step is the following

Proposition 2: Consider system $(a, \epsilon, \mathrm{w}, T)$ and assume that $\mathcal{U}=\epsilon \mathbb{Z}$, then

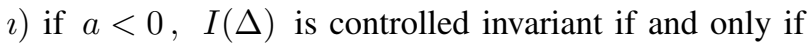

$$
\begin{aligned}
\Delta & \geq \min \left\{\frac{\Gamma \mathrm{w}}{1-\Phi} ; \Gamma(\epsilon+\mathrm{w})\right\}= \\
& =\min \left\{\frac{\mathrm{w}}{|a|} ; \frac{e^{a T}-1}{a}(\epsilon+\mathrm{w})\right\}
\end{aligned}
$$

ı) if $a \geq 0, I(\Delta)$ is controlled invariant if and only if

$$
\begin{aligned}
& \Delta \geq \Gamma(\epsilon+\mathrm{w})=\left\{\begin{array}{lll}
T \cdot(\epsilon+\mathrm{w}) & \text { if } & a=0 \\
\frac{e^{a T}-1}{a}(\epsilon+\mathrm{w}) & \text { if } & a>0 .
\end{array}\right. \\
& \text { Proof: Omitted. }
\end{aligned}
$$

$\mathcal{T}(\Delta)$, and hence the domain of the function $\ell(\Delta, T)$, is determined by solving for $T$ the invariance conditions provided by Proposition 2 :

Corollary 1: Consider system $(a, \epsilon, \mathrm{w}, T)$, assume that $\mathcal{U}=\epsilon \mathbb{Z}$ and let $\Delta>0$. The following facts hold: 
१) if $a<0$ and $\Delta \geq \frac{\mathrm{w}}{|a|}, I(\Delta)$ is controlled invariant $\forall T>0$; if $\Delta<\frac{\mathrm{w}}{|a|}, I(\Delta)$ is controlled invariant if and only if

$$
T \leq \min \left\{\frac{1}{a} \log \left(1-\frac{|a| \Delta}{\epsilon+\mathrm{w}}\right) ; \frac{1}{|a|} \log \left(1+\frac{\mathrm{w}}{\epsilon}\right)\right\} ;
$$

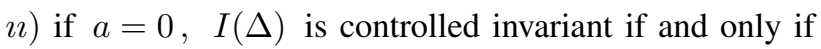

$$
T \leq \frac{\Delta}{\epsilon+\mathrm{w}} ;
$$

ఒu) if $a>0, I(\Delta)$ is controlled invariant if and only if

$$
T \leq \frac{1}{a} \log \left(1+\frac{a \Delta}{\epsilon+\mathrm{w}}\right) .
$$

Before we proceed to the exact calculation of $\ell(\Delta, T)$, we derive a lower bound for $\ell$ from which necessary conditions for the feasibility of a triple $(R, T, \Delta)$ are obtained. To this aim, an invariance criterion holding for systems $(a, \epsilon, \mathrm{w}, T)$ is introduced (more in general, we could assume that $\mathcal{U} \subseteq \mathbb{R}$ ).

Given $\Delta>0, \quad \forall u \in \mathbb{R}$ let $X_{u}:=\left\{x \in \mathbb{R} \mid x^{+}=\right.$ $\Phi x+\Gamma u+w \in I(\Delta), \forall w \in I(\Gamma \cdot \mathrm{w})\}=\{x \in$ $\left.\mathbb{R} \mid-\frac{1}{\Phi}\left(\frac{\Delta}{2}+\Gamma u-\frac{\Gamma \mathrm{w}}{2}\right) \leq x \leq \frac{1}{\Phi}\left(\frac{\Delta}{2}-\Gamma u-\frac{\Gamma \mathrm{w}}{2}\right)\right\}$. Let

$$
\left\{\begin{array}{l}
\alpha_{u}:=-\frac{1}{\Phi}\left(\frac{\Delta}{2}+\Gamma u-\frac{\Gamma \mathrm{w}}{2}\right) \\
\beta_{u}:=\frac{1}{\Phi}\left(\frac{\Delta}{2}-\Gamma u-\frac{\Gamma \mathrm{w}}{2}\right),
\end{array}\right.
$$

if $X_{u} \neq \emptyset$, then $X_{u}=\left[\alpha_{u} ; \beta_{u}\right]$ with measure $\lambda\left(X_{u}\right)=$ $\frac{\Delta-\Gamma \mathrm{w}}{\Phi}$. It is immediate to check that a control set $\mathcal{U} \subseteq \mathbb{R}$ renders $I(\Delta)$ controlled invariant if and only if

$$
\bigcup_{u \in \mathcal{U}} X_{u} \supseteq I(\Delta) \text {. }
$$

A lower bound for $\ell(\Delta, T)$ is provided by the following Lemma 1: Consider system $(a, \epsilon, \mathrm{w}, T)$ and suppose that $\mathcal{U} \subseteq \mathbb{R}$. If $I(\Delta)$ is controlled invariant, then $\# \mathcal{U} \geq$ $\left\lceil\frac{\bar{\Delta} \Phi}{\Delta-\Gamma \mathrm{w}}\right\rceil \geq\lceil\Phi\rceil$.

Proof: By the invariance criterion given in Equation (7) it holds that

$$
\Delta \leq \lambda\left(\bigcup_{u \in \mathcal{U}} X_{u}\right) \leq \# \mathcal{U} \cdot \frac{\Delta-\Gamma \mathrm{w}}{\Phi},
$$

from which the first inequality follows. The second inequality is obvious.

Proposition 3: Consider system $(a, \epsilon, \mathrm{w})$ : necessary conditions for the feasibility of a triple $(R, T, \Delta)$ are

ı) $R \geq \frac{a}{\log 2}+\frac{1}{T} \log _{2} \frac{\Delta}{\Delta-\Gamma(T) \cdot \mathrm{w}}$.

If moreover, $a>0$ :

иi) $T \geq \frac{1}{R}$;

ini) $\Delta \geq \frac{\epsilon+\mathrm{w}}{a}\left(e^{a / R}-1\right)$.

Proof: i) By Theorem 1, $R \geq \frac{1}{T} \log _{2} \ell(\Delta, T) \geq$ $\frac{1}{T} \log _{2} \Phi+\frac{1}{T} \log _{2} \frac{\Delta}{\Delta-\Gamma(T) \cdot \mathrm{w}}=\frac{a}{\log 2}+\frac{1}{T} \log _{2} \frac{\Delta}{\Delta-\Gamma(T) \cdot \mathrm{w}}$ (the second inequality follows by Lemma 1 ).

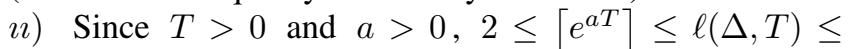
$2^{R T}$ (the second inequality follows by Lemma 1 ).

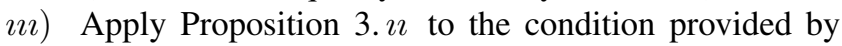
Proposition 2. $\imath$.

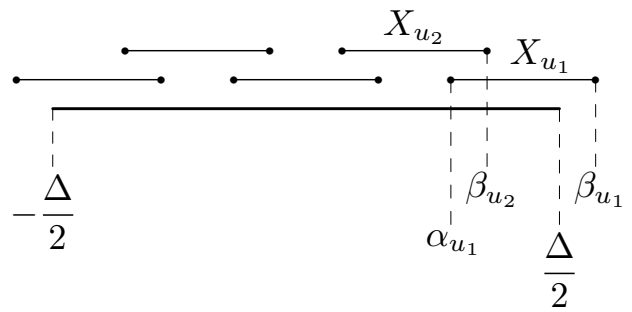

Fig. 2. Construction of $\tilde{\mathcal{U}}$ in the proof of Proposition 4 .

Remark 1: When $\mathrm{w}=0$ and $a>0$, Proposition 3. $\iota$ provides the well-known bound $R \geq \frac{a}{\log 2}$ (see [2]). The same bound is approached when $\Delta \gg \Gamma(T) \cdot \mathrm{w}$.

The condition provided by Proposition 3. $\imath$ shows explicitly that an arbitrarily fast sampling is not allowed.

We pass now to the explicit calculation of the function $\ell(\Delta, T)$ : we will mainly address the case of an unstable plant $(a>0)$, which indeed is the most significant as far as the design of the sampling interval $T$ is concerned.

Proposition 4: Consider system $(a, \epsilon, \mathrm{w}, T)$, assume that $a \geq 0$ and let $\Delta>0$ be such that $T \in \mathcal{T}(\Delta)$. Then

$$
\ell(\Delta, T)=\left\lceil\frac{\Delta / \gamma}{\left\lfloor\frac{\Delta}{\gamma}-\frac{\mathrm{w}}{\epsilon}\right\rfloor} \Phi+\frac{\theta_{1}-\theta_{2}}{\left\lfloor\frac{\Delta}{\gamma}-\frac{\mathrm{w}}{\epsilon}\right\rfloor}\right\rceil:=l,
$$

where $^{1} \gamma=\Gamma \cdot \epsilon, \theta_{1}=\left\lceil\frac{1}{2}\left(\frac{\Delta}{\gamma}(\Phi-1)+\frac{\mathrm{w}}{\epsilon}\right)\right\rceil-\frac{1}{2}\left(\frac{\Delta}{\gamma}(\Phi-\right.$ 1) $\left.+\frac{\mathrm{w}}{\epsilon}\right)$ and $\theta_{2}=\left(\frac{\Delta}{\gamma}-\frac{\mathrm{w}}{\epsilon}\right)-\left\lfloor\frac{\Delta}{\gamma}-\frac{\mathrm{w}}{\epsilon}\right\rfloor$.

Proof: We construct a control set $\tilde{\mathcal{U}} \subset \in \mathbb{Z}$ making $I(\Delta)$ controlled invariant and such that $\# \tilde{\mathcal{U}}=l$. Then we show that a control set $\mathcal{U}_{\text {min }} \subset \epsilon \mathbb{Z}$ of minimal cardinality (with respect to all sets rendering $I(\Delta)$ controlled invariant) is such that $\# \mathcal{U}_{\min }=\# \tilde{\mathcal{U}}$.

To simplify the notation we restrict to the case $\Gamma=\epsilon=1$ and $\mathrm{w}=0$ : the proof in the general case is analogous. Let us construct $\tilde{\mathcal{U}}$ recursively (see Fig. 2), we use the notation introduced in Equation (6):

- Let $\tilde{\mathcal{U}}:=\left\{u_{1}\right\}$ where $u_{1} \in \mathbb{Z}$ is such that

$$
\beta_{u_{1}}=\min _{u \in \mathbb{Z} \text { s.t. } \beta_{u} \geq \frac{\Delta}{2}} \beta_{u}
$$

- While $\alpha_{u_{i}}>-\frac{\Delta}{2}$, let $\tilde{\mathcal{U}}:=\tilde{\mathcal{U}} \cup\left\{u_{i+1}\right\}$ where $u_{i+1} \in \mathbb{Z}$ is such that $\beta_{u_{i+1}}=\min _{u \in \mathbb{Z} \text { s.t. } \beta_{u} \geq \alpha_{u_{i}}} \beta_{u}$.

The algorithm terminates in a finite number of steps because $T \in \mathcal{T}(\Delta)$. By construction, $\tilde{\mathcal{U}}$ satisfies the invariance criterion given by Equation (7). Let us show that $\# \tilde{\mathcal{U}}=l$ : it is easy to verify that $u_{1}=-\left\lceil\frac{1}{2} \Delta(\Phi-1)\right\rceil$, hence we calculate the measure of $X_{u_{1}} \cap I(\Delta)$ which is $\frac{\Delta}{2}-\alpha_{u_{1}}=$ $\frac{\Delta-\theta_{1}}{\Phi}$. The measure of the overlapping of two consecutive intervals associated to the elements of $\tilde{\mathcal{U}}$ is $\beta_{u_{i+1}}-\alpha_{u_{i}}$ : simple calculations show that $u_{i+1}-u_{i}=\lfloor\Delta\rfloor$ and $\beta_{u_{i+1}}-\alpha_{u_{i}}=\frac{\theta_{2}}{\Phi}$. Hence, the cardinality of $\tilde{\mathcal{U}}$ is obtained

${ }^{1}$ The dependence from $T$ is implicit in $\Phi, \gamma, \theta_{1}$, and $\theta_{2}$. 
looking for the smallest integer $s$ such that

$$
\frac{\Delta-\theta_{1}}{\Phi}+(s-1) \frac{\Delta-\theta_{2}}{\Phi} \geq \Delta:
$$

solving the inequality for $s \in \mathbb{R}$ we have $s \geq \frac{\Delta}{\Delta-\theta_{2}} \Phi+$ $\frac{\theta_{1}-\theta_{2}}{\Delta-\theta_{2}}=\frac{\Delta}{\lfloor\Delta\rfloor} \Phi+\frac{\theta_{1}-\theta_{2}}{\lfloor\Delta\rfloor}$; thus the smallest integer satisfying the inequality (9) is $l$.

Let us suppose that $\mathcal{U}_{\text {min }} \subset \mathbb{Z}$ is a control set of minimal cardinality within the family of the sets $\mathcal{U} \subseteq$ $\mathbb{Z}$ rendering $I(\Delta)$ controlled invariant. Suppose that the elements of $\mathcal{U}_{\text {min }}=\left\{u_{1}^{(\min )}, \ldots, u_{m}^{(\min )}\right\}$ are ordered so that $\beta_{u_{1}^{(\min )}}>\beta_{u_{2}^{(\min )}}>\cdots>\beta_{u_{m}^{(\min )}}$. Because of the invariance criterion (7) and by construction of $u_{1}$ it holds that $\beta_{u_{1}^{(\min )}} \geq \beta_{u_{1}}$ : this implies that $\beta_{u_{2}^{(\min )}} \geq \beta_{u_{2}}$ because $\beta_{u_{2}^{(\min )}} \geq \alpha_{u_{1}^{(\min )}} \geq \alpha_{u_{1}}$ and by definition of $\beta_{u_{2}}$. Iterating the same argument it is shown that $\beta_{u_{m}^{(\min )}} \geq \beta_{u_{m}}$ and hence $\alpha_{u_{m}} \leq \alpha_{u_{m}^{(\min )}} \leq-\frac{\Delta}{2}$, since by construction $\alpha_{u_{i}} \leq-\frac{\Delta}{2}$ only for $i=l$ then $l=m=\# \mathcal{U}_{\text {min }}$.

Remark 2: The proof of Proposition 4 also yields an algorithm to construct a control set of minimal cardinality realizing the invariance of $I(\Delta)$.

The formula for $\ell$ given in Equation (8) can be manipulated to obtain a more treatable expression. We shall make use of the following

Lemma 2: $\forall x \in \mathbb{R}$ and $\forall n \in \mathbb{N} \backslash\{0\},\left\lceil\frac{1}{n}\lceil x\rceil\right\rceil=\left\lceil\frac{x}{n}\right\rceil$. Proof: Omitted.

Let $y:=\frac{1}{2}\left(\frac{\Delta}{\gamma}(\Phi-1)+\frac{\mathrm{w}}{\epsilon}\right)$ and plug in Equation (8) the explicit expression for $\theta_{1}$ and $\theta_{2}$, then

$\ell=1+\left\lceil\frac{y+\lceil y\rceil}{\lfloor(\Delta / \gamma)-(\mathrm{w} / \epsilon)\rfloor}\right\rceil=1+\left\lceil\frac{\lceil y+\lceil y\rceil\rceil}{\lfloor(\Delta / \gamma)-(\mathrm{w} / \epsilon)\rfloor}\right\rceil$

by Lemma 2 ; since $\lceil y+\lceil y\rceil\rceil=2\lceil y\rceil$ we get

$$
\ell(\Delta, T)=1+\left\lceil\frac{2\left\lceil\frac{1}{2}\left(\frac{\Delta}{\gamma}(\Phi-1)+\frac{\mathrm{w}}{\epsilon}\right)\right\rceil}{\lfloor(\Delta / \gamma)-(\mathrm{w} / \epsilon)\rfloor}\right\rceil .
$$

Let us make explicit the dependence of $\ell$ from the sampling period $T$ (for $a>0)$ :

$$
\left\{\begin{array}{l}
\ell(\Delta, T)=1+\left\lceil\frac{2\left\lceil\frac{a \Delta+\mathrm{w}}{2 \epsilon}\right\rceil}{\left[\frac{a \Delta}{\epsilon\left(e^{a T}-1\right)}-\frac{\mathrm{w}}{\epsilon}\right]}\right\rceil \\
T \in \mathcal{T}(\Delta) \text { i.e., } \quad 0<T \leq \frac{1}{a} \log \left(1+\frac{a \Delta}{\epsilon+\mathrm{w}}\right) .
\end{array}\right.
$$

B. Determination of $R_{\min }(\Delta)$

We pass now to the determination of the smallest bit rate $R$ ensuring that, for a given $\Delta>0$, there exists a choice of $T$ such that the triple $(R, T, \Delta)$ is feasible. For the sake of brevity, the analysis will henceforth be restricted to the case $a>0$. Let

$$
\begin{gathered}
T_{\max }(\Delta):=\frac{1}{a} \log \left(1+\frac{a \Delta}{\epsilon+\mathrm{w}}\right) . \\
R_{\min }(\Delta):=\min \{R>0 \mid \exists T \text { s.t. }(R, T, \Delta) \text { is feasible }\} .
\end{gathered}
$$

By Theorem 1 and Corollary 1.ıи,

$$
R_{\min }(\Delta)=\min _{\left.T \in] 0 ; T_{\max }(\Delta)\right]} \frac{1}{T}\left\lceil\log _{2} \ell(\Delta, T)\right\rceil .
$$

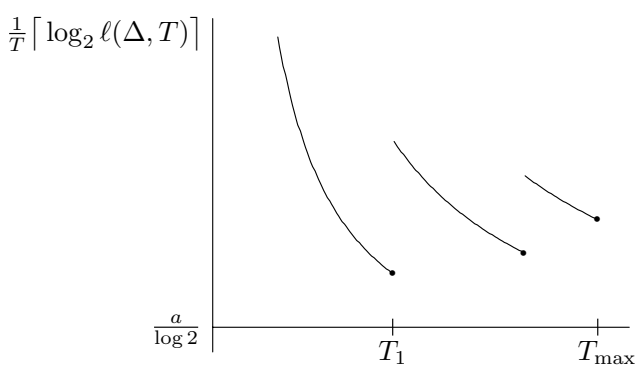

Fig. 3. An example of the graph of $\frac{1}{T}\left\lceil\log _{2} \ell(\Delta, T)\right\rceil$ (for a fixed $\Delta$ ).

Given $\Delta>0$, the mapping $\ell(\Delta, T)$ is piecewise constant with $T$. Hence, the local minima of the "channel occupation" function $\frac{1}{T}\left\lceil\log _{2} \ell(\Delta, T)\right\rceil$ are taken in correspondence of discontinuity points of $\ell(\Delta, T)$. The discontinuity points $T_{1}<T_{2}<\cdots<T_{k}$ can be determined using Equation (11), thus the local minima of the channel occupation function can be listed. However, a closed formula for $R_{\min }(\Delta)$ is difficult to work out. We hence provide an expression which is a good estimate of $R_{\min }(\Delta)$.

Proposition 5: Consider system $(a, \epsilon, \mathrm{w})$ and assume that $a>0$. For $\Delta>0$, a sufficient condition on $R$ in order that the triple $(R, T, \Delta)$ is feasible for some $T>0$ is

$$
R \geq R_{\min }^{\text {suf }}(\Delta):=\frac{a}{\log \left(1+\frac{a \Delta}{2 \epsilon\left\lceil\frac{a \Delta+\mathrm{w}}{2 \epsilon}\right\rceil+\mathrm{w}}\right)} .
$$

Proof: Indeed $R_{\min }^{\text {suf }}(\Delta)=\frac{1}{T_{1}}\left\lceil\log _{2} \ell\left(\Delta, T_{1}\right)\right\rceil$, where $T_{1}$ is the first discontinuity point of $\ell(\Delta, T)$. Let us calculate $T_{1}$ : for a given $\Delta>0$, the argument of the floor in the denominator of Equation (11) is a decreasing function of $T$, in particular $\ell(\Delta, T)$ is non-decreasing with $T$. To determine $T_{1}$ it is sufficient to find the largest $T$ such that the denominator in Equation (11) is greater than or equal to the numerator, that is to solve

$$
\frac{a \Delta}{\epsilon\left(e^{a T}-1\right)}-\frac{\mathrm{w}}{\epsilon}=2\left\lceil\frac{a \Delta+\mathrm{w}}{2 \epsilon}\right\rceil \text {. }
$$

Hence,

$$
T_{1}=\frac{1}{a} \log \left(1+\frac{a \Delta}{2 \epsilon\left\lceil\frac{a \Delta+\mathrm{w}}{2 \epsilon}\right\rceil+\mathrm{w}}\right)
$$

and

$$
\left.\ell(\Delta, T)=2 \quad \forall T \in] 0 ; T_{1}\right] .
$$

The thesis follows.

Remark 3: The effect of the noise on the system is governed by the function $\Gamma(T)$ which grows exponentially: hence, as the sampling interval $T$ increases, the system is more and more affected by the noise. It is then natural to expect that the local minimum in correspondence of the first discontinuity point $T_{1}$ is close to the actual minimum of the function (see Fig. 3). This fact can be explicitly verified for $a \Delta \gg \max \{\epsilon, \mathrm{w}\}$, in fact (see also Fig. 4):

$$
\lim _{\Delta \rightarrow+\infty} R_{\min }^{\text {suf }}(\Delta)=\frac{a}{\log 2},
$$




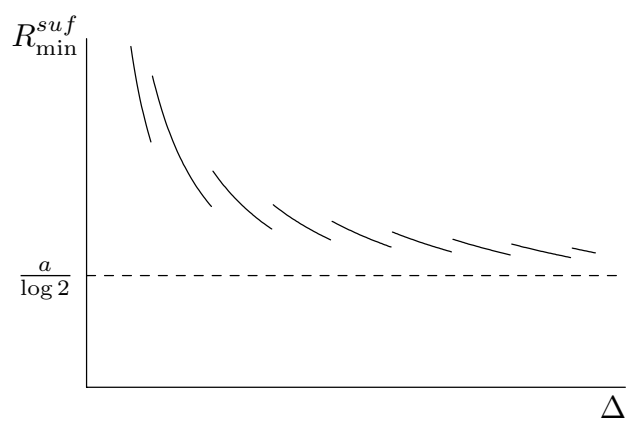

Fig. 4. The graph of $R_{\min }^{\text {suf }}(\Delta)$ for the system of Fig. 3.

namely, as $\Delta \rightarrow+\infty, R_{\min }^{\text {suf }}(\Delta)$ approaches the theoretical lower bound on $R$ (see Proposition 3. 2 and Remark 1). Notice also that the feasible triple $\left(R_{\min }^{\text {suf }}(\Delta), T_{1}, \Delta\right)$ leads to the implementation of a binary control law.

Remark 4: For a given $\Delta>0$, if $R$ is not much larger than $R_{\min }(\Delta)$, the set of values of $T$ such that $(R, T, \Delta)$ is feasible consists of disjoint intervals whose right extremes are discontinuity points of the function $\ell(\Delta, T)$ (see Fig. 3). A criterion to discriminate the allowed values for $T$, apart from robustness arguments, should take into account of the entailed channel occupation $\frac{1}{T}\left\lceil\log _{2} \ell(\Delta, T)\right\rceil$. Remark 5 (Coping with transmission-delay): In this paper we have assumed that the control selected by the controller is instantaneously applied by the actuator. This is apparently unrealistic in presence of a finite capacity channel. However, the analysis presented thus far can be adapted to cope with transmission delay if the controller is endowed with memory (so that at the instant $k$ the controller knows the current state and the control values applied at time $k-1$ ).

\section{CONCLUSIONS AND FUTURE WORK}

In this paper we gave the basic theoretical tools to produce automated design procedures for control design of systems with quantized actuators and bit rate constraints. Particular emphasis was put on the presence of noise that exposes important aspects of the system's behavior. Many interesting points remains open for future investigations. It is already in progress a similar analysis for transient behaviors [3], when the state has to be attracted into $I(\Delta)$ from an enclosing region, and also an efficient algorithm for solving problem (5) has been devised (to appear elsewhere). Finally, a further goal is the study of event-based sampling a la Lebesgue [1] which is almost naturally induced by the presence of quantization. However, it is not evident under which conditions such a sampling could lead to significant advantages in terms of average required bit rate.

\section{APPENDIX}

Proof: [Proposition 1] Let $\tilde{x}_{m}(t)$ be the solution of

$$
\left\{\begin{array}{l}
\dot{\tilde{x}}(t)=a \tilde{x}(t)+u-\frac{\mathrm{w}}{2} \\
\tilde{x}(0)=x^{0}
\end{array}\right.
$$

and $\tilde{x}_{M}(t)$ be the solution of the same system with $+\frac{\mathrm{w}}{2}$ in place of $-\frac{\mathrm{w}}{2}$. For any integrable function $\tilde{w}:[0 ; T] \rightarrow$ $I(\mathrm{w})$ the solution $\tilde{x}(t)$ of system (3) is such that $\tilde{x}_{m}(t) \leq$ $\tilde{x}(t) \leq \tilde{x}_{M}(t)$, in fact:

$$
\tilde{x}(t)-\tilde{x}_{m}(t)=\int_{0}^{t} e^{a(t-s)}\left(\tilde{w}(s)+\frac{\mathrm{w}}{2}\right) d s \geq 0
$$

because the integrand is positive. The other inequality is analogous. It is then sufficient to show that $\forall t \in$ $[0 ; T], \quad \tilde{x}_{m}(t) \in I(\Delta)$ and $\tilde{x}_{M}(t) \in I(\Delta)$. By hypothesis, $\tilde{x}_{m}(T) \in I(\Delta)$. Since $\tilde{x}_{m}(t)$ is the solution of the differential equation $\dot{\tilde{x}}=a \tilde{x}+u-\frac{\mathrm{w}}{2}=f(\tilde{x})$ not depending explicitly on $t$, then it is a monotonic function and the thesis consequently follows. The same holds for $\tilde{x}_{M}(t)$.

\section{REFERENCES}

[1] K.J. Åström and B. Bernhardsson, Comparison of Riemann and Lebesgue sampling for first order stochastic systems, Proc. of the 41st IEEE Conf. on Decision and Control, pages: 2011-2016. 2002.

[2] J. Baillieul, Feedback designs in information-based control, in Proc. of the Workshop on Stochastic Theory and Control, Kansas, 2001, pages: 35-57, Springer-Verlag.

[3] A. Bicchi, K.H. Johansson, L. Palopoli and B. Picasso, Quantised control in distributed embedded systems, in Proc. of the Int. Symp. on Math. Theory of Networks and Systems, Leuven (B), 2004.

[4] F. Blanchini, Set Invariance in Control, Automatica, 35; pages: 17471767. 1999.

[5] R. Brockett, Minimum Attention Control, Proc. of the 36th IEEE Conf. on Decision and Control, pages: 2628-2632. 1997.

[6] R. Brockett and D. Liberzon, Quantized feedback stabilization of linear systems, IEEE Trans. Autom. Control, 45(7); pages: 12791289. 2000

[7] A. Cervin, J. Eker, B. Bernhardsson and K.E. Arzen, FeedbackFeedforward Scheduling of Control Tasks, Real-Time Systems 1(23). 2002.

[8] D.F. Delchamps, Stabilizing a Linear System with Quantized State Feedback, IEEE Trans. Autom. Control, 35(8); pages: 916-924. 1990.

[9] N. Elia and S. Mitter, Stabilization of Linear Systems With Limited Information, IEEE Trans. Autom. Control, 46(9); pages: 1384-1400. 2001.

[10] F. Fagnani and S. Zampieri Stability analysis and synthesis for scalar linear systems with a quantized feedback, IEEE Trans. Autom. Control, 48; pages: 1569-1584. 2003.

[11] F. Fagnani and S. Zampieri, Quantized stabilization of linear systems: complexity versus performance, to appear on Trans. Autom. Control, special issue on "Networked Control Systems". 2004.

[12] D. Hristu and K. Moransen, Limited Communication Control, System and Control Letters 37(4); pages: 193-205. 1999.

[13] H. Ishii and B.A. Francis, Stabilizing a Linear Systems by Switching Control With Duel Time, IEEE Trans. Autom. Control, 47(12); pages: 1962-1973. 2002.

[14] D. Liberzon, Hybrid feedback stabilization of systems with quantized signals, Automatica, 39; pages: 1543-1554. 2003.

[15] G.N. Nair and R.J. Evans, Exponential stabilisability of finitedimensional linear systems with limited data rates, Automatica, 39; pages: 585-593. 2003.

[16] L. Palopoli, Design of Embedded Control Systems under real-time scheduling constraints, Ph.D. thesis, ReTiS Lab - Scuola Superiore S. Anna - Pisa. 2002.

[17] B. Picasso, F. Gouaisbaut and A. Bicchi, Construction of invariant and attractive sets for quantized-input linear systems, Proc. of the 41st IEEE Conf. on Decision and Control, pages: 824-829. 2002.

[18] B. Picasso and A. Bicchi, On the Stabilization of Linear Systems Under Assigned I/O Quantization, submitted. 2004.

[19] S.C. Tatikonda, Control under communication constraints, Ph.D. thesis, Massachusetts Institute of Technology. 2000.

[20] W. Wong and R. Brockett, Systems with finite communication bandwidth constraints - part II: Stabilization with limited information feedback, IEEE Trans. Autom. Control, 44(5); pages: 1049-1053. 1999. 